Ann. Zootech., I977, 26 (2), 255-27I.

\title{
Effets de l'habitat et de l'origine génétique sur les performances d'engraissement et la précocité sexuelle de jeunes truies de race Large White
}

\author{
G. BOLET, M. ÉTIENNE $(*)$ et C. LEGAULT \\ avec la collaboration technique de J. Gruand, G. Conseil et Christiane Vachot \\ Station de Génétique Quantitative et Appliquée, \\ (*) Station de Recherches sur l'Elevage des Porcs, \\ Centre national de Recherches zootechniques, I.N.R.A., \\ $7^{8} 350$ Jouy-en-Josas (France)
}

\section{Résumé}

224 jeunes truies issues de deux populations, le troupeau expérimental Large White de La Minière et la population Large White du Poitou, ont été comparées à partir de $30 \mathrm{~kg}$ suivant un schéma factoriel $2 \times 2$ dans đeux milieux, en alimentation à volonté : une porcherie de La Minière (Yvelines), de type fermé, en loges de 5 femelles et la porcherie du C.E.S.P. à Rouillé (Vienne), de type semi-plein air, par loges de 10. A $80 \mathrm{~kg}$, on a calculé pour chaque animal la vitesse de croissance et l'indice de consommation entre 30 et $80 \mathrm{~kg}$, l'épaisseur de lard (mesurée par ultra sons) à $80 \mathrm{~kg}$. Le contrôle de puberté s'est déroulé individuellement jusqu'à l'âge maximum de 300 jours; les jeunes truies étaient saillies à la première chaleur et abattues 30 jours après, afin de dénombrer les corps jaunes et les embryons vivants.

Pour l'ensemble des performances d'engraissement et pour la précocité sexuelle, l'effet du milieu d'élevage est prépondérant. Les jeunes truies élevées à Rouillé présentent une meilleure vitesse de croissance, une meilleure efficacité alimentaire, mais une plus grande adiposité; elles atteignent la puberté plus jeunes et à un poids moins élevé, mais présentent un taux d'ovulation et un nombre d'embryons vivants à 30 jours plus faibles que celles élevées à I a Minière.

Les truies du Poitou atteignent $80 \mathrm{~kg}$ plus tard que celle originaires de La Minière; ceci est dû à une moins bonne vitesse de croissance entre 30 et $80 \mathrm{~kg}$ (à Rouillé), mais aussi à un retard de croissance pris avant $30 \mathrm{~kg}$, période où l'on ne peut pas distinguer l'effet du milieu et de l'origine génétique. Par contre, à $80 \mathrm{~kg}$ comme à l'abattage, elles manifestent une moindre adiposité; elles atteignent la puberté à un poids significativement plus faible et à un âge légèrement plus faible, dû essentiellement à une réduction de l'intervalle de temps $30 \mathrm{~kg}$ - puberté. L'effet du milieu semble dû essentiellement à une liaison importante entre la vitesse de croissance et la précocité sexuelle; il semble également que les conditions de la croissance avant zo kg puissent avoir un effet non négligeable sur les performances ultérieures et sur le déclenchement de la puberté. 


\section{Introduction}

La période précédant la mise à la reproduction d'une jeune truie constitue une phase improductive dont l'allongement a une incidence économique défavorable (LEGAULT et DAGORN, I973). Or l'âge à la puberté est le facteur limitant d'une mise à la reproduction précoce; en race Large White, il est de l'ordre de $2 \mathrm{I}_{4}$ jours, avec des fluctuations annuelles comprises entre 202 et 24I jours (LEGAULT, I973); on peut considérer comme retardée toute jeune truie dont la première chaleur survient au-delà de 240 jours. Les causes des retards à la puberté qui frappent en général un élevage dans son ensemble peuvent être nombreuses (MARTINAT et al., I970). Elles sont d'autant plus difficiles à déterminer que milieu (alimentation, habitat, conduite de l'élevage...) et type génétique sont en général confondus.

Dans cet esprit, la Commission Porcine de l'I.N.R.A. a proposé la mise en place d'une expérience permettant d'analyser les effets de l'habitat, du type génétique et leurs interactions éventuelles sur la précocité sexuelle de jeunes truies, ainsi que les relations avec les performances d'engraissement de ces animaux. Nous présentons ici les résultats de cette étude.

\section{Matériel et méthodes}

\section{I. - Matériel animal}

Les jeunes truies mises en contrôle proviennent de deux populations :

- le troupeau Large White de la Station de Recherches sur l'Élevage des Porcs, situé à La Minière (Yvelines); 86 jeunes truies sont issues de I4 verrats et de 40 femelles de ce troupeau;

- la population Large White du Poitou; I62 jeunes truies sont issues de I3 verrats ayant été contrôlés individuellement au Centre Expérimental de Sélection Porcine et mis en service à la Station Expérimentale d'Insémination Artificielle de Rouillé (Vienne), et de 42 mères se trouvant réparties dans des élevages du Poitou (I à 5 pour chaque verrat).

\section{2. - Conditions expérimentales}

A un poids vif compris entre 20 et $25 \mathrm{~kg}$, les jeunes femelles des deux populations, nées en août I975, ont été réparties dans les deux milieux suivants :

- Une porcherie de La Minière, de type fermé. Les animaux sont placés en I3 loges de 5 animaux ( 9 loges de femelles originaires de La Minière et 4 loges de femelles criginaires du Poitou).

- La porcherie du Centre Expérimental de Sélection Porcine de Rouillé (Vienne), de type semi-plein air. Les femelles sont réparties à raison de ro par loge; dans les deux milieux, les loges sont affectées au hasard à chaque origine génétique et peuplées d'animaux de poids comparable pour pouvoir analyser l'indice de consommation moyen de la loge.

Dans les deux élevages, les loges comportent une aire de couchage et une aire de déjection, la surface totale par porc étant de $2,75 \mathrm{~m}^{2}$ à La Minière et de $\mathrm{I}, \mathrm{I} 3 \mathrm{~m}^{2}$ 
à Rouillé. Les sœurs de portée, ont été, dans la mesure du possible, réparties également entre ces deux milieux.

Les jeunes truies sont nourries à volonté (à l'auge à La Minière, au nourrisseur automatique à Rouillé) à partir du poids moyen de $30 \mathrm{~kg}$ avec le même aliment sans antibiotiques présenté sous forme de granulés et fourni pour les deux milieux par le même fabricant : type "croissance " ( $16 \%$ de matières azotées et 3 roo kcal d'énergie digestible par $\mathrm{kg}$ ) jusqu'au poids moyen de la loge de $60 \mathrm{~kg}$, type "finition" ( 4 \% de matières azotées et 3 Ioo kcal d'énergie par kg) de $60 \mathrm{~kg}$ à l'abattage.

Les conditions de température relevées pendant l'expérience sont rapportées dans la figure $x$. Les mesures ont été effectuées à l'extérieur de la porcherie en semiplein air de Rouillé, et à l'intérieur du bâtiment fermé de La Minière.

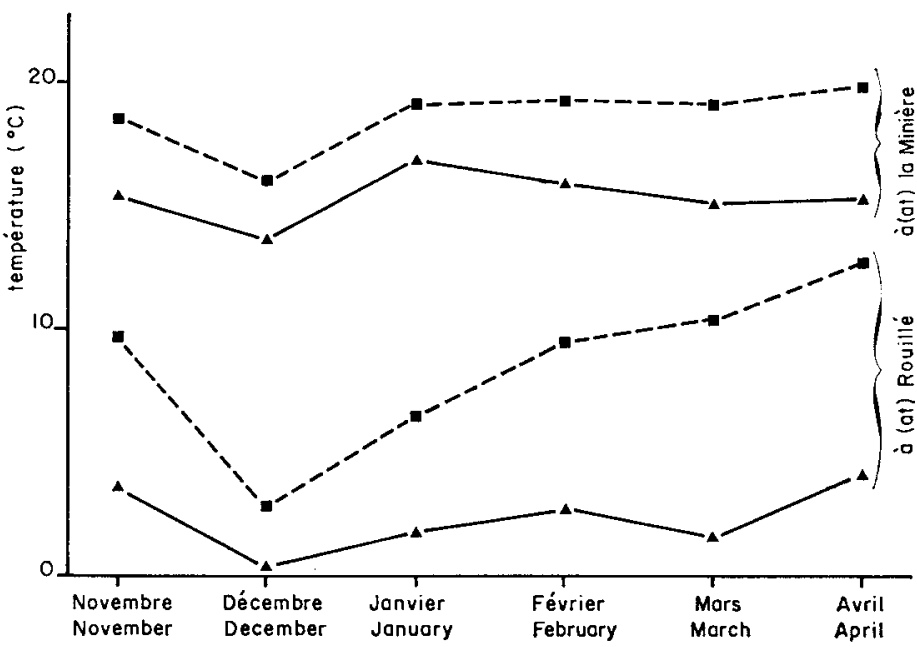

FIG. I. - Conditions de température au cours de l'étude $\left({ }^{\circ} \mathrm{C}\right)$

Temperature conditions during the experiment, in ${ }^{\circ} \mathrm{C}$

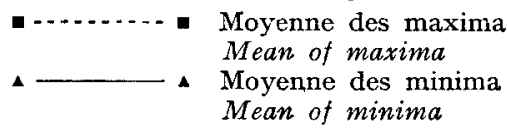

\section{3. - Modalités des contrôles}

Les truies sont pesées toutes les deux semaines, ainsi qu'à la puberté et le jour de l'abattage; pour chaque truie, l'épaisseur du lard dorsal est mesurée à un poids aussi proche que possible de $80 \mathrm{~kg}$ avec un appareil à ultra sons. La valeur retenue pour l'analyse est la moyenne de six mesures (au niveau du cou, du dos et du rein) et corrigée par régression linéaire pour les variations de poids vif $(0,2 \mathrm{~mm} / \mathrm{kg})$.

L'indice de consommation est mesuré par loge à partir du début du contrôle (30 kg) jusqu'à $80 \mathrm{~kg}$ de poids moyen par loge. La valeur moyenne obtenue est affectée à chaque animal de la loge.

Le contrôles des chaleurs a lieu pour chaque loge chaque matin dès qu'un animal atteint $80 \mathrm{~kg}$ de poids vif, à l'aie d'udn verrat boute-en-train, jusqu âl'âge de 300 jours. Les femelles sont inséminées artificiellement à Rouillé, et saillies par 
un verrat à La Minière, le jour de la puberté et le lendemain. Les jeunes truies sont abattues à $30 \pm 3$ jours de gestation lırsqu'elles ont été saillies, et à 300 jours d'âge quand elles sont encore impubères; l'abattage a lieu une fois par semaine à l'abattoir du C.N.R.Z. à Jouy-en-Josas pour les truies élevées à La Minière, aux établissements Archaimbault à Celle-sur-Belle (Deux-Sèvres) pour celles élevées à Rouillé. Au cours de cette opération sont relevés le poids vif de l'animal, le poids net sans tête et l'épaisseur du lard dorsal au niveau de la dernière vertèbre lombaire (rein), de la dernière vertèbre dorsale (dos) et de la dernière vertèbre cervicale (cou).

Parmi les mesures effectuées sur le tractus génital prélevé au moment de l'abattage, nous avons retenu pour cette analyse le nombre de corps jaunes disséqués et le nombre d'embryons vivants à 30 jours.

\section{4. - Analyse statistique}

L'expérience répond à un dispositif factoriel $2 \times 2$, à savoir deux types génétiques et deux milieux.

Pour chaque variable étudiée, nous avons soumis les données à une analyse de variance à effets fixés par la méthode des moindres carrés (HARVEY, I960); le modèle général utilisé est le suivant :

$$
\mathrm{Y}_{i j k}=\mu+r_{i}+m_{j}+(r m)_{i j}+{ }_{1} \sum \beta^{(1)} \mathrm{X}_{i j k}^{(1)}+\mathrm{E}_{i j k}
$$

ou

$\mu$ représente la moyenne générale

$r_{i}$ l'effet de l'origine génétique $(i=\mathrm{I}$ ou 2$)$

$m_{j}$ l'effet du milieu d'élevage ( $j=\mathrm{I}$ ou 2 )

$(r m)_{i j}$ le terme d'interaction origine génétique $\times$ milieu (4 niveaux)

$\mathrm{X}_{i j k}^{(1)}$ la covariable affectée du coefficient de régression linéaire $\beta$ (1) de la variable étudiée $\mathrm{Y}_{i j k}$ sur $\mathrm{X}_{i j k}^{(\mathrm{I})}$.

Les $\mathrm{E}_{i j k}$ sont des variables aléatoires résiduelles supposées normales indépendantes, d'espérance nulle et de variance $\sigma^{2}$.

Les covariables utilisées suivant les variables étudiées seront précisées dans la présentation des résultats.

Cette analyse permet d'obtenir les tests de Frscher sur les effets principaux et les interactions, les estimées des moindres carrés attachées à chaque niveau des effets principaux et de l'interaction, ainsi que les corrélations résiduelles entre les variables étudiées avec un même modèle.

Pour préciser la signification d'interactions éventuelles, nous avons également comparé 2 à 2 à l'aide de tests de STudent les quatre niveaux du terme d'interaction origine génétique $\times$ milieu.

\section{Résultats}

\section{I. - Caractères d'engraissement}

Les effectifs de truies sont rapportés dans le tableau I. Au total, 24 truies ont été éliminées au cours du contrôle d'engraissement pour accidents ou raisons sanitaires.

Le contrôle a débuté au poids moyen de $30,2 \mathrm{~kg}$ et s'est terminé au poids moyen de $80,8 \mathrm{~kg}$. Nous avons analysé l'âge au début et à la fin du contrôle (âge 
TABLEAU I

Répartition des effectifs des jeunes trites au cours des contróles d'engraissement et de puberté

Distribution of gilts during fattening and puberty controls

\begin{tabular}{|c|c|c|c|c|c|}
\hline $\begin{array}{c}\text { Milieu } \\
\text { Environment }\end{array}$ & \multicolumn{2}{|c|}{ La Minière } & \multicolumn{2}{|c|}{ Rouillé } & \multirow{2}{*}{ Total } \\
\hline $\begin{array}{l}\text { Origine génétique } \\
\text { Genetic stock }\end{array}$ & I a Minière & Poitou & La Minière & Poitou & \\
\hline $\begin{array}{l}\text { Effectif : au début du contrôle }(30 \mathrm{~kg}) \\
\text { Number of females at } 30 \mathrm{~kg}\end{array}$ & 43 & 20 & 43 & 142 & $24^{8}$ \\
\hline 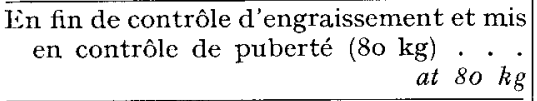 & $4^{\circ}$ & 16 & $4^{2}$ & 126 & 224 \\
\hline $\begin{array}{l}\text { Ayant termine le contrôle de puberté } \\
\text { Number at the end of the puberty control }\end{array}$ & 38 & 16 & $4^{2}$ & I I9 & $2 \mathrm{I}_{5}$ \\
\hline Total $\cdot \cdot \cdot \cdot \cdot \cdot \cdot \cdot \cdot \cdot$ & $3^{8}$ & I5 & 39 & $\begin{array}{r}109(*) \\
71 \quad\left(3^{8}\right) \\
\end{array}$ & $20 I$ \\
\hline 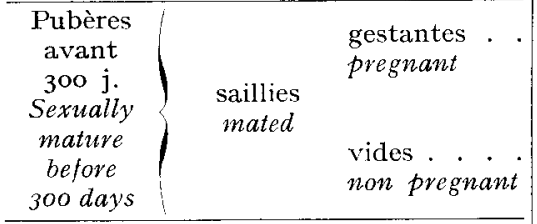 & 25 & 9 & 23 & $44(22)$ & IOI $\left(\begin{array}{lll}1 & 23\end{array}\right)$ \\
\hline \begin{tabular}{c|l} 
Impubères & $\begin{array}{l}\text { Impubères à } 300 \mathrm{j} . \\
\text { before } 300 \text { days }\end{array}$ \\
Sexually & Fausses chaleurs (abat- \\
immatuve & $\begin{array}{l}\text { tues avant } 300 \mathrm{j} .) . \\
\text { Heat withoutovulation }\end{array}$ \\
(slaughtered before 300 days)
\end{tabular} & o & o & 2 & 8 & I I \\
\hline
\end{tabular}

(*) Parmi les truies du Poitou élevées à Rouillé, 38 ont été gardées dans le cadre d'une expérience de sélection. Vingt deux ont été gestantes à la première saillie (qui a lieu à la ${ }^{\mathrm{re}}$ ou à la $2^{\mathrm{e}}$ chaleur), $\mathbf{I} 6$ ont eu un retour en chaleur. Thirty-eight of the gilts born and maintained in Poitou were kept in a selection experiment. Twenty-two were pregnant at first service (effected at first or second heat), 16 returned to oestrus.

à $30 \mathrm{~kg}$ et âge à $80 \mathrm{~kg}$ ), le gain moyen quotidien entre 30 et $80 \mathrm{~kg}$ (GMQ 30-80), l'épaisseur de lard à $80 \mathrm{~kg}$ et l'indice de consommation moyen entre 30 et $80 \mathrm{~kg}$. Les résultats de l'analyse de variance pour l'ensemble des animaux ayant atteint la fin du contrôle sont rassemblés dans le tableau 2.

L'effet de l'origine des jeunes truies est significatif pour l'âge à $30 \mathrm{~kg}$ et à $80 \mathrm{~kg}(\mathrm{P}<0,005)$, l'épaisseur de lard $(\mathrm{P}<0,005)$. Les animaux originaires du Poitou sont plus âgés à $30 \mathrm{~kg}(+8 \pm 2$ jours) et à $80 \mathrm{~kg}(+\mathrm{Io} \pm 3$ jours), mais sont moins gras $(-3 \pm 0,5 \mathrm{~mm})$ que ceux originaires de La Minière. L'effet du milieu est significatif $(\mathrm{P}<0,005)$ pour toutes les variables sauf l'âge à $30 \mathrm{~kg}$; les truies élevées à Rouillé ont une meilleure vitesse de croissance $(+\mathrm{I} 36 \pm$ I6 $\mathrm{g} / \mathrm{j}$ ) et un meilleur indice de consommation $(-0,42 \pm 0,02)$, mais sont plus grasses $(+3,4 \pm 0,5 \mathrm{~mm})$ que celles élevées dans la porcherie de La Minière. La figure 2 fait apparaître une interaction origine $\times$ milieu significative pour le GMQ $30-80 \mathrm{~kg}(\mathrm{P}<0$,OI $)$ et l'indice de consommation $(\mathrm{P}<0,05)$ : dans la porcherie de Rouillé, les animaux originaires de La Minière ont une vitesse de crois- 
TABLEAU 2

Résultats des tests de " $F$ "et estimées des moindres carrés pour les caractères d'engraissement jusqu'à $80 \mathrm{~kg}$

" $F$ " values and least squares estimates for fattening performance before $80 \mathrm{~kg}$

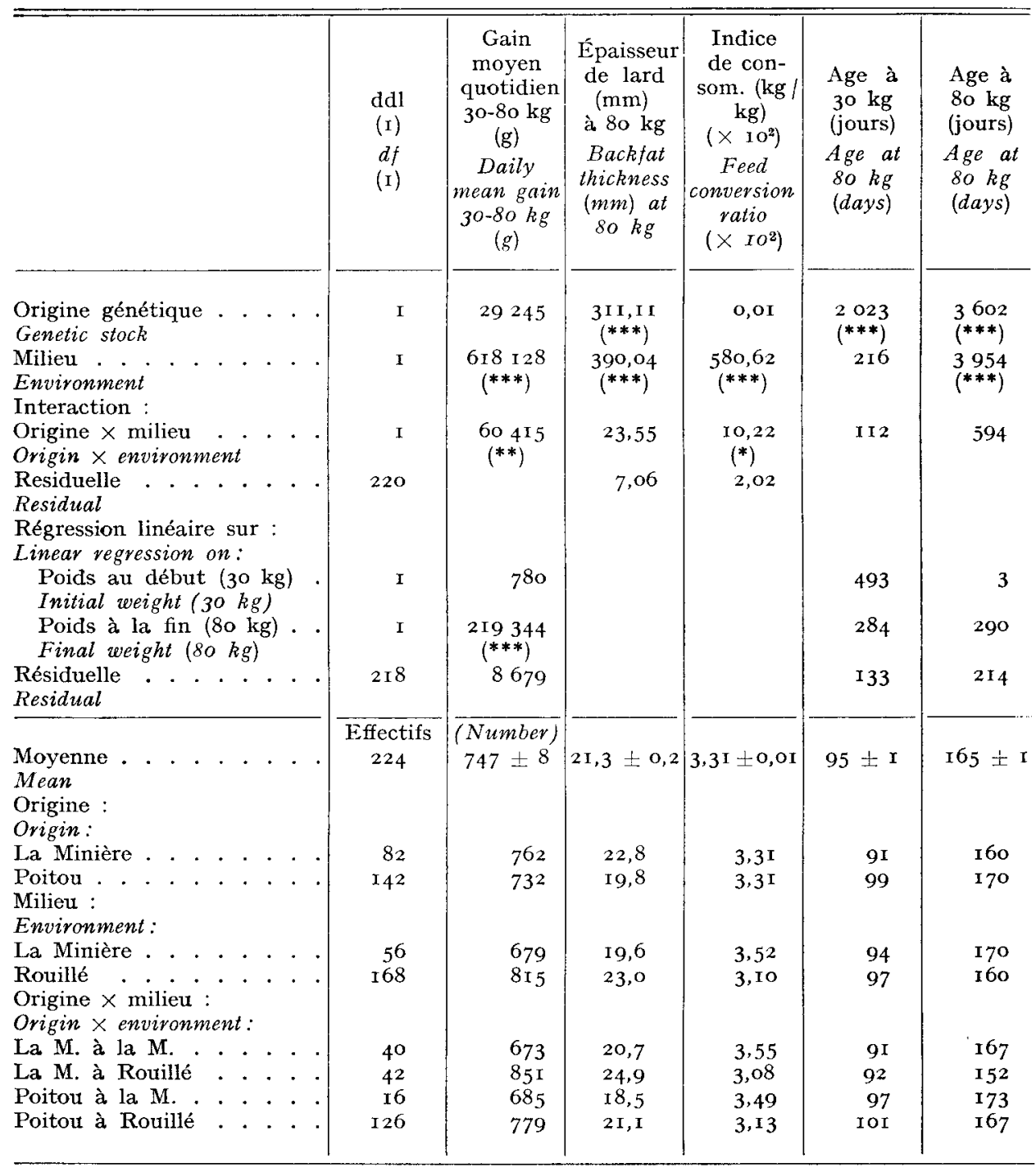

*** $\mathrm{P}<0,005 \quad$ ** $\mathrm{P}<\mathrm{0}, \mathrm{or} \quad * \mathrm{P}<0,05$

(I) ddl $=$ degrés de liberté; $d f=$ degree of freedom.

sance nettement plus élevée $(+72 \pm \mathrm{I} 7 \mathrm{~g} / \mathrm{j})$ et un indice de consommation plus faible $(-0,05 \pm 0,03)$ que ceux originaires du Poitou. A l'inverse, un avantage non significatif de ces derniers se manifeste à La Minière pour la vitesse de croissance $(+\mathrm{I} 3 \pm 28 \mathrm{~g} / \mathrm{j})$ et l'indice de consommation $(-0,06 \pm 0,04)$ (fig. I). On peut comparer les quatre groupes de truies grâce à un index économique 

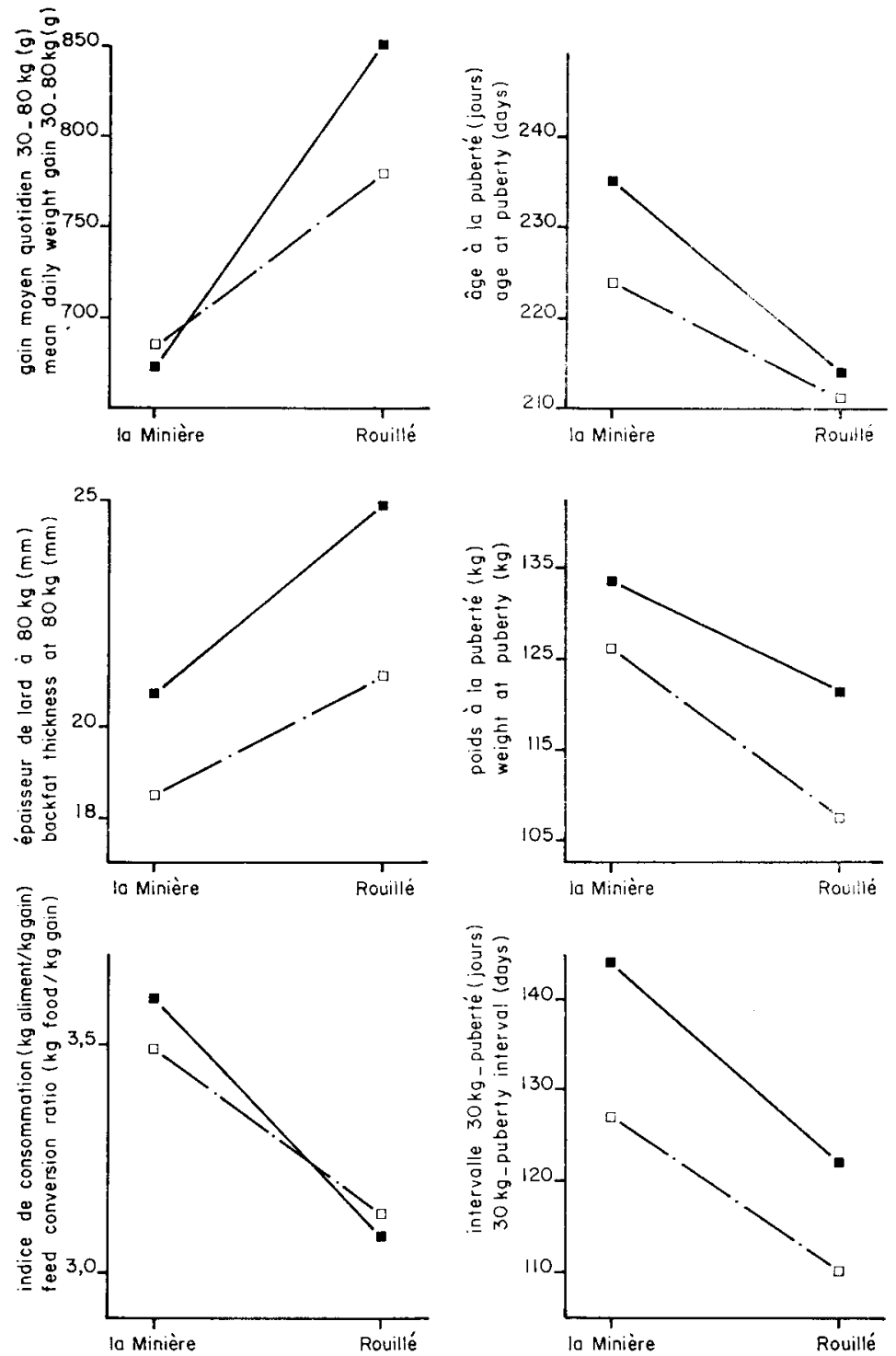

FIG. 2. - Effets du changement de milieu sur les performances d'engraissement et de 'reproduction Environmental effects on fattening and reproductive performance

- Truies originaires de La Minière

Gilts born in La Minière

[-_._- - Truies originaires du Poitou

Gills born in Poiton

pour la période d'engraissement $30-80 \mathrm{~kg}$ calqué sur celui utilisé entre 20 et $100 \mathrm{~kg}$ dansles stations de contrôle individuel (Molenat, Houix et Poulenc, I974), exprimé en francs.

$$
\mathrm{I}=\mathrm{o}_{2} \mathrm{I}(\mathrm{GMQ}-\overline{\mathrm{GMQ}})-5 \mathrm{O}(\mathrm{IC}-\overline{\mathrm{IC}})-5(\mathrm{Ep} . \text { lard }-\overline{\mathrm{Ep} .} \text { Lard })
$$




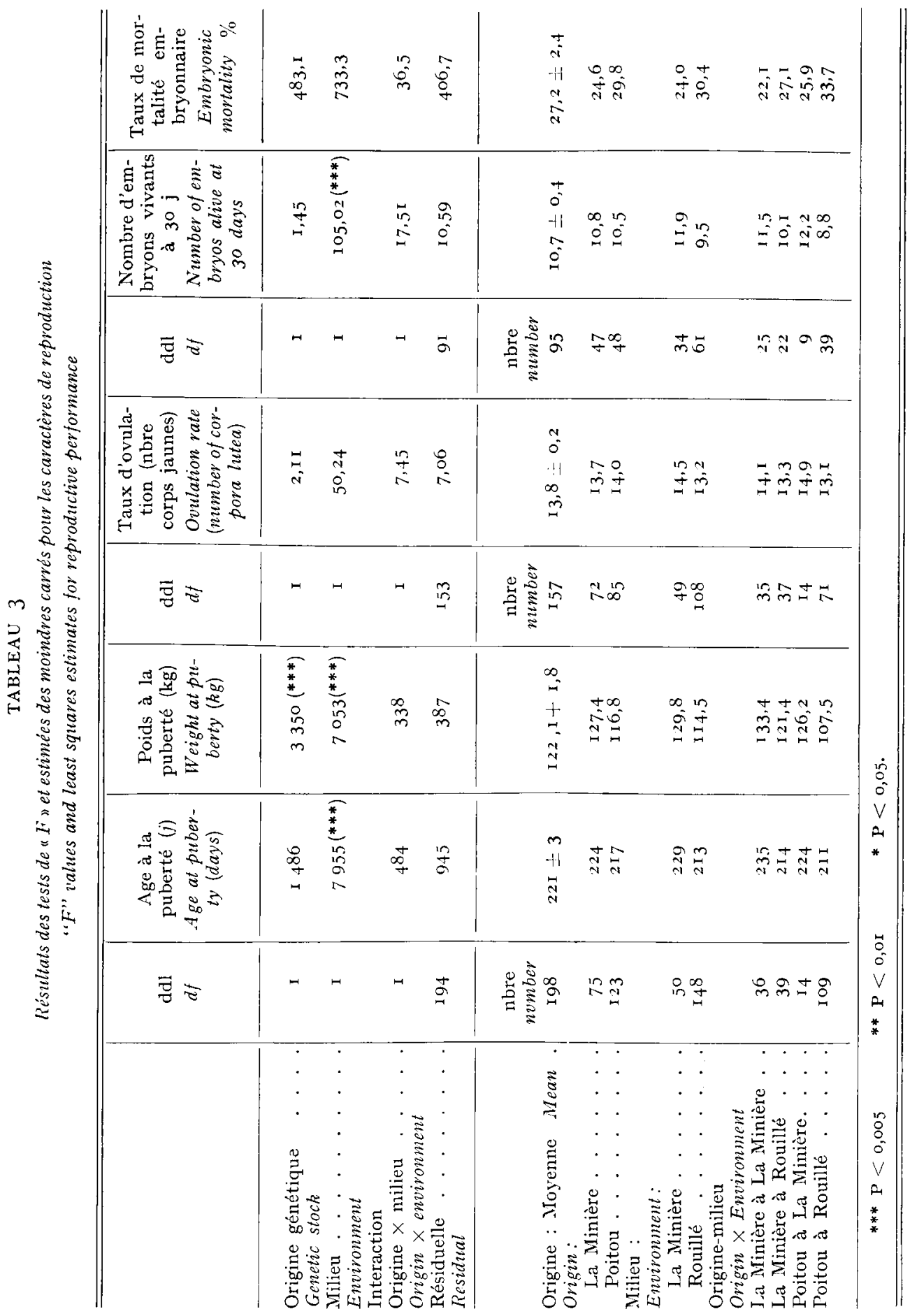


( $\mathbf{g}$ de GMQ supplémentaire vaut o, Io Franc, un point d'indice de consommation coûte $50 \mathrm{~F}$ et I mm de lard en plus coûte $5 \mathrm{~F}$ ). On obtient les valeurs suivantes :

$\begin{array}{cll}\text { Truies originaires de : } & \text { La Minière élevées à La Minière : - I6,07 F } \\ & \text { La Minière } & \text { Rouillé }:+3,44 \mathrm{~F} \\ & \text { Rouillé } & \text { La Minière }:-\mathrm{r}, 26 \mathrm{~F} \\ & \text { Rouillé } & \text { Rouillé }:+13,78 \mathrm{~F}\end{array}$

Les deux types génétiques ont une meilleure valeur économique quand ils sont élevés à Rouillé; à l'intérieur de chaque milieu d'engraissement, il se manifeste une supériorité de valeur économique des truies issues du Poitou par rapport à celles originaires de La Minière.

\section{2. - Caractères de reproduction}

Neuf truies ont été éliminées au cours du contrôle de puberté pour accidents ou raisons sanitaires.

On n'observe aucun effet significatif du milieu d'élevage $\left(\hat{\chi}_{1}^{2}=\mathrm{I}, 64\right)$ ou de l'origine génétique des jeunes truies $\left(\hat{\chi}_{1}^{2}=\mathrm{I}, 8 \mathrm{I}\right)$ sur le pourcentage de truies pubères à 300 jours (par rapport au nombre de truies ayant terminé le contrôle de puberté), ni sur le pourcentage de truies pleines à la première saillie $\left(\hat{\chi}_{1}^{2}=0,32\right.$ pour l'effet du milieu et $0,7 \mathrm{I}$ pour l'effet de l'origine génétique). Les effectifs sont rassemblés dans le tableau $I$.

L'analyse de variance (tabl. 3) révèle un effet significatif du milieu pour toutes les variables sauf le taux de mortalité embryonnaire. Les animaux élevés à Rouillé atteignent la puberté plus jeunes (- I $6 \pm 6$ jours) à un poids moins élevé $(-15,3$ $\pm 3,6 \mathrm{~kg}$ ); leur taux d'ovulation est moins élevé ( $-\mathrm{I}, 3 \pm 0,5$ corps jaunes) ainsi que le nombre d'embryons vivants à 30 jours pour les truies gestantes $(-2,4$ $\pm 0,8)$. L'origine génétique des animaux exerce un effet significatif sur leur poids à la puberté, plus faible pour les truies originaires du Poitou (- I $0,6 \pm 3,6 \mathrm{~kg})$. On ne note aucune interaction significative entre l'origine génétique et le milieu d'élevage. Par contre, il apparaît un effet significatif à la fois du milieu $(\mathrm{P}<0,005)$ et de l'origine génétique $(\mathrm{P}<0,05)$ sur l'intervalle de temps entre le début du contrôle à $30 \mathrm{~kg}$ et la puberté : il est plus faible à la porcherie de Rouillé qu'à celle de La Minière ( $-\mathbf{I} 8 \pm 3$ jours) et pour les animaux du Poitou que pour ceux issus de La Minière (- I $3 \pm 3$ jours).

\section{3. - Caractéristiques de la carcasse}

A poids vif constant à l'abattage, l'origine génétique a un effet très significatif $(\mathrm{P}<0,005)$ sur le rendement et l'épaisseur de lard aux trois sites de mesures. Les truies originaires de La Minière ont un rendement plus élevé $\left(7^{8} \%\right.$ contre $76 \%$ ); pour chaque milieu d'élevage, elles présentent une adiposité plus grande que les truies originaires du Poitou. A Rouillé, où l'on a mesuré la longueur de la carcasse entre l'atlas et le bord antérieur de la symphyse pubienne, on ne note aucune différence significative entre types génétiques pour ce caractère (tabl. 4). 
TABLEAU 4

Tests de " $F$ " et estimées des moindres carrés pour les mesures sur la carcasse " $F$ " values and least squars estimates for carcass measurements

\begin{tabular}{|c|c|c|c|c|c|c|}
\hline & \multirow{2}{*}{$\begin{array}{l}\mathrm{ddl} \\
d f\end{array}$} & \multirow{2}{*}{$\begin{array}{c}\text { Age abat. } \\
\text { (j) } \\
\text { Age at } \\
\text { slaughter } \\
\text { (days) }\end{array}$} & \multirow{2}{*}{$\begin{array}{c}\text { Poids net } \\
(\mathrm{kg}) \\
\text { Net weight } \\
(\mathrm{kg})\end{array}$} & \multicolumn{3}{|c|}{$\begin{array}{l}\text { Épaisseur de lard (mm) } \\
\text { Mean backfat thickness }\end{array}$} \\
\hline & & & & $\begin{array}{l}\text { Rein } \\
\text { Loin }\end{array}$ & $\begin{array}{c}\text { dos } \\
\text { Back }\end{array}$ & $\begin{array}{c}\text { cou } \\
\text { Neck }\end{array}$ \\
\hline $\begin{array}{l}\text { Origine } \\
\text { Origin }\end{array} \cdot \ldots \cdot \ldots$ & I & 36 & $\begin{array}{l}70,5 \\
(* * *)\end{array}$ & $\begin{array}{l}965,7 \\
(* * *)\end{array}$ & $\begin{array}{l}487,4 \\
(* * *)\end{array}$ & $\begin{array}{l}\text { I } 657,7 \\
(* * *)\end{array}$ \\
\hline $\begin{array}{l}\text { Milieu . . . . . . } \\
\text { Environment }\end{array}$ & 1 & 103 & I 7,3 & 24,5 & 7,6 & $57, \mathrm{I}$ \\
\hline $\begin{array}{l}\text { Origine } \times \text { milieu } \\
\text { Origin } \times \text { envivonment } \\
\text { Covariable }=\text { ooids à l'abat. }\end{array}$ & I & 2258 & 0,3 & 22,8 & 1,0 & $\begin{array}{l}280,8 \\
(*)\end{array}$ \\
\hline 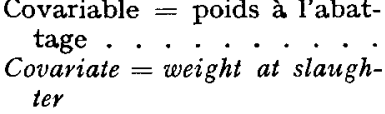 & 1 & $\begin{array}{l}\text { I5 } 5 \text { 610 } \\
(* * *)\end{array}$ & $\begin{array}{l}27939,9 \\
(* * *)\end{array}$ & $\begin{array}{l}2322,5 \\
(* * *)\end{array}$ & $\begin{array}{l}\text { I } 934,0 \\
(* * *)\end{array}$ & $\underset{(* * *)}{3 \underset{6}{9} 6,3}$ \\
\hline $\begin{array}{l}\text { Résiduelle } \cdot \ldots \cdot \ldots \cdot \cdot \cdot \\
\text { Residual }\end{array}$ & I 30 & 709 & $\mathrm{I} 7,4$ & $4^{6,2}$ & 30,8 & 54,6 \\
\hline $\begin{array}{l}\text { Moyenne } . . . . . . . . ~ \\
\text { Mean } \\
\text { Origine : } \\
\text { Origin: } \\
\end{array}$ & Effectifs & $25 I=4$ & $108,5 \pm 0,6$ & $39, \mathbf{I} \pm 0,9$ & $34,8 \pm 0,8$ & $57,8 \pm 1,0$ \\
\hline La Minière . . . . . & $5^{8}$ & 250 & I IO, I & 43,0 & 37,6 & 62,9 \\
\hline $\begin{array}{l}\quad \text { Poitou . . . . } \cdot \\
\text { Milieu : } \\
\text { Environment: }\end{array}$ & 77 & 252 & I06,8 & 35,2 & 32,0 & $5^{2,6}$ \\
\hline La Minière & 27 & 252 & I O9, I & 38,4 & 34,4 & 56,7 \\
\hline Rouillé & 108 & 249 & 107,9 & 39,8 & 35,2 & 58,9 \\
\hline La Minière à La Minière & $2 \mathrm{I}$ & 258 & 110,7 & 42,9 & 37,0 & 64,0 \\
\hline La Minière à Rouillé . . & 37 & 243 & 109,6 & 43,2 & $3^{8, I}$ & 61,9 \\
\hline Poitou à La Minière & 6 & 247 & 107,5 & 33,8 & 31,7 & 49,4 \\
\hline $\begin{array}{c}\text { Poitou à Rouillé } \\
\beta(\mathbf{I}) \\
\end{array}$ & $7 \mathrm{I}$ & $\begin{array}{r}256 \\
+\quad 0,6\end{array}$ & $\begin{aligned} & 106,2 \\
+ & 0,8\end{aligned}$ & $\begin{array}{l}36,5 \\
+0,2\end{array}$ & $\begin{array}{r}32,3 \\
+\quad 0,2\end{array}$ & $\begin{array}{r}55,8 \\
+\quad 0,3\end{array}$ \\
\hline
\end{tabular}

*** $\mathrm{P}<0,005 \quad$ ** $\mathrm{P}<0$, OI * $\mathrm{P}<0,05$.

(I) $\beta=$ coefficient de régression linéaire. $\beta=$ Linear regression coefficient.

\section{4. - Corrélations résiduelles}

Les corrélations entre performances d'engraissement et de reproduction sont rassemblées dans le tableau 5. Elles sont significatives entre la vitesse de croissance et l'âge à la puberté d'une part $(-0,28)$, le poids à la puberté d'autre part $(0,18)$. Il apparait également une corrélation élevée entre l'âge et le poids à la puberté $(0,66)$ et entre le taux d'ovulation et le nombre d'embryons vivants à 30 jours $(0,48)$.

\section{Discussion et conclusion}

Le principal intérêt de cette étude est de dissocier l'effet de l'habitat et du type génétique sur la précocité sexuelle des deux troupeaux qui présentent généralement des écarts importants pour l'âge à la puberté : plus de 240 jours en moyenne 
TABLEAU 5

Corrélations entre caractères d'engraissement et de reproduction Correlations between fattening and reproductive traits

\begin{tabular}{|c|c|c|c|c|}
\hline $\begin{array}{c}(n=\text { nombre } \\
\text { d'observations }) \\
(n=\text { number of } \\
\text { observations })\end{array}$ & $\begin{array}{l}\text { Poids puberté } \\
(n=198) \\
\text { Weight at } \\
\text { puberty }\end{array}$ & $\begin{array}{c}\text { Age puberté } \\
\left(n=19^{8}\right) \\
\text { Age at } \\
\text { puberty }\end{array}$ & $\begin{array}{c}\text { Taux d'ovulation } \\
(n=157) \\
\text { Onulation rate }\end{array}$ & $\begin{array}{l}\text { Nombre d'em- } \\
\text { bryons vivants } \\
\text { vivants } \\
(n=95) \\
\text { Number of em- } \\
\text { bryos alive }\end{array}$ \\
\hline $\begin{array}{l}\text { Gain moyen quotidien } \\
30-80 \mathrm{~kg} . \\
\text { Growth rate } 30-80 \mathrm{~kg} \\
\text { Epaisseur lard } 80 \mathrm{~kg} . \\
\text { Backfat thickness at } 80 \mathrm{~kg} \\
\text { Age à la puberté . . } \\
\text { Age at puberty } \\
\text { Poids à la puberté . . } \\
\text { Weight at puberty } \\
\text { Taux d'ovulation . . } \\
\text { Ovulation vate }\end{array}$ & $\begin{array}{l}0,18 \quad(*) \\
0,02 \\
0,66 \quad(* *)\end{array}$ & $\begin{array}{l}-0,28(* *) \\
-0,0_{4}\end{array}$ & $\begin{array}{l}0,08 \\
0,01 \\
0,01 \\
0,12\end{array}$ & $\begin{array}{l}0,10 \\
-0,07 \\
0,07 \\
0,18 \\
0,48 \quad(* *)\end{array}$ \\
\hline${ }^{* *} \mathrm{P}<0$, or & ${ }^{*} \mathrm{P}<0,05$. & & & \\
\hline
\end{tabular}

pour le troupeau de La Minière, 2I4 jours (moyenne sur 9 années) pour les truies du Poitou mises en contrôle au C.E.S.P. à Rouillé. Il faut cependant remarquer que les animaux n'ayant été transférés de leur lieu de naissance au lieu d'étude qu'après avoir atteint $20 \mathrm{~kg}$ de poids vif, l'effet génétique se trouve confondu avec les influences des conditions antérieures de milieu, et avec les conséquences éventuelles des regroupements et transports effectués; mais ce biais paraît difficile à éviter. Les animaux étudiés dans le présent travail, issus d'un nombre assez élevé de verrats et de portées, peuvent être considérés comme suffisamment représentatifs des deux populations comparées; on notera cependant le faible effectif de truies du Poitou élevées à La Minière ( 16 en fin de contrôle). Pour les caractères d'engraissement et de précocité sexuelle, l'analyse porte sur la totalité des animaux ayant terminé les contrôles. Par contre, les analyses pour les autres caractères portent sur des effectifs plus réduits : en particulier, 38 truies du Poitou élevées à Rouillé ont été replacées chez des éleveurs dans le cadre d'un programme de sélection sur les performances d'engraissement en place au C.E.S.P.; ceci provoque une troncature importante dans l'échantillon, car elles présentent une vitesse de croissance supérieure de $5^{\mathrm{I}} \mathrm{g}$ et une épaisseur de lard inférieure de I,6 $\mathrm{mm}$ à la moyenne des $7 \mathrm{I}$ autres truies du Poitou pubères abattues.

Pour diverses raisons expérimentales, les embryons vivants à 30 jours n'ont été dénombrés que sur 95 truies et les mesures de carcasse effectuées sur I35 animaux; les résultats concernant ces deux dernières mesures doivent donc être considérés avec une certaine réserve.

\section{I. - Caractères d'engraissement}

Jusqu'au poids de $30 \mathrm{~kg}$, les facteurs milieu et origine génétique sont confondus : toutes les truies du troupeau de La Minière sont élevées dès leur naissance et nourries de façon rationnelle en porcherie fermée, alors que les porcelets du 
Poitou sont dispersés dans de nombreuses fermes jusqu'à $20-25 \mathrm{~kg}$, où l'on peut penser qu'ils ne bénéficient pas toujours d'une alimentation optimale pendant cette phase. De plus, le choix a été plus sévère pour les animaux de La Minière puisque parmi un nombre de portées comparable, 86 jeunes femelles ont été gardées au lieu de 162 pour celles du Poitou. Ceci apparaît également à travers la proportion d'animaux ne terminant pas les contrôles de croissance, qui est de 4,7 p. Ioo pour les truies provenant de Ia Minière, contre 12,3 p. Ioo pour les autres. Tout cela pourrait expliquer que les animaux du Poitou ont une moins bonne vitesse de croissance jusqu'à $30 \mathrm{~kg}$, sans que l'on sache s'il s'agit d'un effet du milieu, de l'origine génétique ou des deux facteurs à la fois.

Entre 30 et $80 \mathrm{~kg}$, le principal facteur de variation des performances d'engraissement est le milieu d'élevage : l'élevage cn semi-plein air à Rouillé permet une meilleure vitesse de croissance, une meilleure efficacité alimentaire, mais aussi une plus forte adiposité. A régime alimentaire strictement identique, le type d'habitat semble donc avoir un effet important sur les performances d'engraissement; ce résultat est en accord avec l'étude de Blancharv, TARDIF et EngRAnd (I976) réalisée sur I 2I ooo porcs et montrant essentiellement l'influence du volume d'air disponible par porc sur les caractères de croissance et de carcasse. Par ailleurs, Diggs, Baker et Rogers (1965) et SPRouse et al. (1975) notent une croissance plus rapide des animaux élevés en plein air par rapport à leurs contemporains maintenus en claustration. A l'inverse, WAHLSTROM, Fredrikson et Libal (I97I) n'observent aucune différence entre ces deux types d'habitat. JENSEN et al. (I969) trouvent, pour la vitesse de croissance, un effet légèrement favorable (surtout en début de croissance) de l'engraissement en hiver dans une porcherie fermée par rapport à un bâtiment de semi-plein air. Les truies originaires de La Minière manifestent une moilleure vitesse de croissance mais aussi une plus forte adiposité que celles du Poitou. Mais la différence entre les deux origines génétiques est beaucoup plus marquée à Rouillé qu'à La Minière (fig. I); les animaux de La Minière, possédant le meilleur potentiel de croissance, ne semblent pas pouvoir exprimer leur supériorité dans le milieu le moins favorable, et sont plus sensibles que ceux du Poitou au changement de porcherie.

En fait, aucun des deux milieux et aucune des deux origines génétiques n'associent à la fois la meilleure vitesse de croissance et la plus faible adiposité (la corrélation entre le GMQ 30-80 kg et l'épaisseur de lard à $80 \mathrm{~kg}$ est $0,2 \mathrm{I}$ ). Les résultats concernant l'efficacité alimentaire font apparaître la supériorité du milieu de Rouillé par rapport à celui de La Minière. Ceci peut difficilement être relié au type d'habitat proprement dit, la plupart des travaux réalisés montrant que l'élevage des animaux en plein air ou en semi-plein air augmente l'indice de consommation par rapport à leur maintien en claustration (SEYMour et al., rg64; DigGs, BAKER et Rogers, I965; Jensen et al., I969). Un moins bon état sanitaire des animaux, lié à l'absence de vide sanitaire strict, pourrait expliquer les plus mauvais résultats observés à La Minière. Cependant, les taux d'élimination des animaux avant la fin des contrôles de croissance (II,I \% contre $9,2 \%$ à Rouillé) ne font pas apparaître de différence notable.

Les écarts importants pour l'indice économique sont dus essentiellement aux variations de l'indice de consommation entre les milieux comparés. Toutefois, cet indice ne rend pas compte des variations économiques associées aux fluctuations du rendement à l'abattage, plus favorable aux femelles originaires de La Minière. 


\section{2. - Précocité sexuelle}

Le pourcentage de truies pubères à 300 jours, 93,5 p. Ioo, est sensiblement égal aux valeurs obtenues généralement en race Large White; par contre, le taux de non retour en chaleur à 30 jours comparable dans les deux milieux $(64,7 \%)$ est anormalement faible. LEGAult (I973) obtient, sur 6 ans, une moyenne de 90,4 p. Ioo pour le taux de puberté à 300 jours et 80,2 p. Ioo pour le taux de réussite en insémination artificielle. Le nombre de retours en chaleur observés peut être attribué en partie aux températures estivales survenues très tôt en I976; on observe classiquement une chute du taux de réussite en été (Du Mesnil Du Buisson et Signoret, I973). L'avantage de 4 p. Ioo à La Minière, avec saillie naturelle, par rapport à Rouillé, avec insémination artificielle, n'est pas significatif.

Le principal facteur de variation de la précocité sexuelle est le milieu: l'âge et le poids à la puberté sont significativement plus faibles à Rouillé qu'à La Minière; les valeurs pour ces deux caractères à Rouillé concordent très bien avec celles déjà relevées dans ce milieu en race Large White (LEGAULT, I973; RousseaU, I975); les truies issues de La Minière sont pubères au même âge que celles du Poitou $(+3 \pm 6$ jours) mais sont significativement plus lourdes $(+13,9 \pm 3,7 \mathrm{~kg})$. A la porcherie de La Minière, les truies du Poitou sont plus précoces (- II -t Io jours) et moins lourdes $(-7,2 \pm 6,2 \mathrm{~kg})$ mais ces différences ne sorst pas significatives; l'âge moyen de 229 jours est nettement inférieur à celui généralement relevé (en moyenne supérieur à 240 jours) dans cet élevage. C'est en particulier le cas de I9 truies contemporaines des animaux de cette expérience et destinés à la reproduction, maintenues dans une autre porcherie de La Minière, et dont l'àge à la puberté a été de 252 jours. Dans le cadre d'études sur la nutrition des jeunes femelles avant la puberté réalisées dans cet élevage, la maturité sexuelle est atteinte tardivement: pour les lots les plus précoces, elle est de 296 jours (ÉTIENNE et DUEE, I973) ou 255 jours (DUEE, I977). Ces moins bons résultats pourraient en partie s'expliquer par le fait que les animaux étaient rationnés, contrairement à ceux de la présente expérience. En effet, d'après Haines, Warnick et Wallace (i959), ZimmerMAN et al. (I960) ou FRIEND (I976), une restriction alimentaire sévère retarde la puberté des jeunes truies. Toutefois, dans le cas d'un rationnement moins drastique il y aurait peu ou pas d'effet sur la précocité sexuelle de femelles (LoDce et Mac Pherson, Ig6r).

Peut-on expliquer la plus grande précocité sexuelle à Rouillé par la différence de mode d'habitat? Un certain nombre d'auteurs mettent en évidence l'effet favorable de l'élevage en plein air sur le déclenchement de la puberté (MeAcham et Massincup i970; Salmon-Legagneur, I970). Jensen et al. (I970) observent une différence significative entre truies attachées et truies élevées en groupe, mais pas entre celles élevées en enclos ou dans un local à $33-35^{\circ} \mathrm{C}$. Dans la présente étude, les conditions de milieu comparées diffèrent essentiellement par les températures relevées, l'écart étant de l'ordre de Io ${ }^{\circ} \mathrm{C}$, et les variations importantes à Rouillé se trouvant tamponnées à La Minière. En fait, le milieu semble agir essentiellement sur l'âge à la puberté par le biais de la vitesse de croissance : les truies élevées à Rouillé ont la croissance la plus rapide et sont les plus précoces sexuellement, tant pour l'âge que pour le poids. Ceci est confirmé par les corrélations significatives que l'on trouve entre la vitesse de croissance et l'âge à la puberté $(-0,28)$ et surtout entre la vitesse de croissance (GMQ 30-80) et l'intervalle de temps $30 \mathrm{~kg}$ - puberté $(-0,25)$. L'intervalle $30 \mathrm{~kg}$ - puberté, sur lequel on peut dissocier l'effet du milieu et de l'origine génétique, est significativement réduit pour 
les jeunes truies du Poitou ( $-\mathrm{I} 3 \pm 3$ jours); il semble y avoir compensation du retard pris avant $30 \mathrm{~kg}$ (la corrélation entre l'âge à $30 \mathrm{~kg}$ et la durée $30 \mathrm{~kg}$ - puberté est de $-0,19)$. Mais cet avantage des truies du Poitou se réduit à $-7 \pm 6$ jours pour l'âge à la puberté et n'est pas significatif. Il semblerait donc que la croissance avant $30 \mathrm{~kg}$ exerce une certaine influence sur la précocité sexuelle, ce qui est confirmé par la corrélation de $0, \mathrm{I} 8$ entre l'âge à $30 \mathrm{~kg}$ et l'âge à la puberté. Mais ce résultat n'est pas tout à fait en accord avec les observations de NELSON et RoBISoN (1976) selon lesquelles une réduction du poids des jeunes truies à l'âge de 8 semaines n'a pas de conséquence sur l'apparition de la première chaleur. Quoi qu'il en soit, nos résultats vont dans le même sens que ceux de Reutzel et SumpTION (I968) et ANDERSON et MELAMPy (I972) qui observent que, intra lignée, les truies à meilleure vitesse de croissance sont plus précoces mais que les lignées ayant la meilleure croissance tendent à être pubères plus tard.

Notons que nous ne trouvons aucune corrélation significative entre la précocité sexuelle et l'adiposité. Les différentes corrélations que nous obtenons entre performances d'engraissement et de reproduction concordent parfaitement avec celles obtenues par Reutzel et Sumption (I968) et Cunningham et al. (I974) sur les jelnes truies du "gene pool " de l'université du Nebraska.

\section{3. -- Taux d'ovulation et nombre d'embryons vivants à 30 jours.}

On n'observe pas d'effet de l'origine génétique sur le taux d'ovulation, mais un effet du milieu important (- I,2 corps jaune à Rouillé); on peut remarquer que le taux d'ovulation est plus élevé dans le milieu où les truies sont les plus âgées et les plus lourdes à la puberté. En fait, on ne trouve pas de corrélation entre l'âge à la puberté et le taux d'ovulation. Au contraire, si on ramène le taux d'ovulation à poids constant, on obtient une corrélation de - 0,09 entre l'âge et le nombre de corps jaunes, semblant indiquer que les truies les plus précoces ovulent davantage; ces résultats concordent avec ceux de LEGAULT (I973) et RoussEAU (I975); de même O'Bannon et al. (I966), Étienne et Duee (I973), Young, Omtvedt et Johnson (I974) et DUEE (I977) ne trouvent pas de corrélation significative entre âge à la puberté et taux d'ovulation, et une corrélation positive entre poids à la puberté et taux d'ovulation. Par contre Reddy, Lasley et Mayer (I958), Lerner et al. (I957) observent une liaison positive entre l'âge à la puberté et le taux d'ovulation.

Nous ne mettons pas en évidence de corrélations significatives entre le nombre de corps jaunes ou d'embryons vivants et les performances d'engraissement, ce qui est en accord avec l'hypothèse que les performances d'élevage sont pratiquement indépendantes des caractères de croissance et de carcasse (LEGAULT, I97I).

L'effet du milieu sur le nombre d'embryons vivants à 30 jours est également très significatif; ce nombre est supérieur de 2,4 à La Minière. Ceci pourıait être dû en partie à un effet défavorable de l'insémination artificielle par rapport à la saillie naturelle. SKJERVOLD (I975) note de même une diminution de la taille de la portée en insémination artificielle; il pense que l'on ne doit pas l'attribuer à un taux d'ovulation plus faible, mais à une moins bonne fertilisation des ovules ou à une mortalité embryonnaire accrue. Il faut noter que les truies gestantes ont un taux d'ovulation supérieur de 0,9 à la moyenne des truies abattues. La corrélation entre taux d'ovulation et nombre d'embryons vivants $(0,48)$ est élevée et 
supérieure à celles généralement observées (LEGAULT, I973). Les valeurs obtenues dans cette expérience pour le taux d'ovulation et le nombre d'embryons à Rouillé sont sensiblement égales à celles observées en moyenne sur 9 ans (Rousseau, r975), mais elles sont exceptionnellement élevées à La Minière pour des truies nullipares saillies à la première chaleur.

En définitive, dans les conditions où elle a été réalisée, cette étude nous a permis de mettre en évidence l'effet prépondérant du milieu sur les performances d'engraissement et la précocité sexuelle : les truies élevées à Rouillé présentent une meilleure vitesse de croissance, une meilleure efficacité alimentaire, mais une plus forte adiposité; elles sont plus précoces sexuellement mais ont un taux d'ovulation plus faible. Nous n'avons pas mis en évidence d'effet très marqué de l'origine génétique sauf pour l'adiposité (plus élevée pour les truies de La Minière); la meilleure vitesse de croissance des truies de La Minière et la plus grande précocité sexuelle de celles du Poitou ne sont pas très nettes; en fait, les truies du Poitou semblent avoir pris du retard pour la croissance pendant la phase précédant la mise en contrôle, ce qui aurait eu un effet défavorable sur le déclenchement de la puberté.

Reçu pour publication en mars 1977.

\section{Summary}

\section{Environmental and genetic effects on fattening performance and sexual maturity of Large White gilts}

A total of 224 gilts from 2 different populations, the Large White experimental herd of La Minière and the Large White population of the Poitou region, were fed ad libitum. The animals were compared in a $2 \times 2$ factorial design in two environments: a confined heated building in La Minière (78-FRANCE) with pens containing 5 females, and an open-front house in Rouillé (86-FRANCE) with to pigs per pen. Daily mean gain and feed efficiency were determined between 30 and $80 \mathrm{~kg}$, and backfat thickness was ultrasonically measured at $80 \mathrm{~kg}$ for each animal. The ostrus control began at $80 \mathrm{~kg}$ and was stopped at a maximum age of 300 days. The gilts were mated at the first ostrus and slaughtered 30 days after service in order to measure the number of corpora lutea and of live embryos.

The environmental effect was preponderant for growth performance and sexual precocity. The gilts raised at Rouillé had a faster growth rate, a better feed efficiency, and were fatter. They reached puberty younger and at a lower body weight, but their ovulation rate and the number of embryos alive at 30 days of pregnancy were lower than those of females raised at La Minière.

The gilts born in Poitou reached the weight of $80 \mathrm{~kg}$ later than those born in La Miniere. This was due to the lower growth rate of the former between $30 \mathrm{~kg}$ and $80 \mathrm{~kg}$, and to a growth delay before $30 \mathrm{~kg}$, period during which the environmental and genetic effects could not be separated. On the other hand, the gilts born in Poitou showed a lower fatness at $80 \mathrm{~kg}$ and at slaughter, reached puberty at a significantly lower weight and at an age which tended to be lower, essentially in relation with a reduction of the time interval between $30 \mathrm{~kg}$ and puberty. The environmental effect seemed to be essentially due to an important relationship between growth rate and sexual maturity. It seemed also that growth conditions before $30 \mathrm{~kg}$ could be involved in subsequent performances and puberty.

\section{Références bibliographiques}

Anderson L. L., Melampy R. M., 1972. Factors affecting ovulation rate in the pig. In Pig Production, 329-366, D.J.A. Cole, Butterworths (London).

Blanchard D., Tardif H., Engrand E., 1976. Contribution à l'étude de l'influence de certains facteurs du milieu. sur les performances des porcs à l'engrais. Journées Rech. Porcine en France, 335-344, INRA, ITP Ed., Paris. 
Cunningham P. J., Naber C. H., Zimmerman D. R., Peo E. R., 1974. Influence of nutritional regime on age at puberty in gilts. J, Anim. Sci., 39, 63-67.

Diggs B. G., Baker B. Jr., Rogers R. W., I965. Influence of protein level and confined feeding on performance and carcass characteristics of swine. J. Anim. Sci., 24, 283 (Abstr.).

DÚ́E P. H., 1977. Influence d'une restriction azotée, à partir de $25 \mathrm{~kg}$ de poids vif, sur les performances de reproduction de la truie. Journées Rech. Porcine en France, INRA, ITP Ed., Paris, 193-198.

Étienne M., Duee P. H., 1973. Effets respectifs des niveaux alimentaires pendant la croissance et le premier mois de gestation sur les performances de reproduction chez la truie multipare : résultats préliminaires, Ann. Zootech., 22, 453-462.

Friend D. W., 1976. Nutritional effects on age at puberty and plasma amino acid level in Yorkshire gilts and on chemical composition, nucleic acid, fatty acid and hydroxyproline contents of the uterus. J. Anim. Sci., 43, 404-4Iz.

Haines C. E., Warnick A. C., A. Wallace H. D., i959. The effects of two levels of energy intake on reproductive phenomena in Duroc Jersey gilts. J. Anim. Sci., $18,355-360$.

HARVEY W. R., 1960. Least squares analysis of data with unequal subclass numbers. A.R.S. 20-8, Biom. Serv., A.R.S., U.S.D.A., Washington D.C. ed.

Jensen A. H., Kuhlman D.E., Becker D. E., Harmon B. G., I969. Response of growingfinishing swine to different housing environments during winter seasons. J. Anim. Sci., 29, $451-456$.

Jensen A. H., Yen J. T., Gehring M. M., Baker D. E., Becker D. E., Harmon B. G., i97o. Effects of space restriction and management on pre-and post-puberal response of female swine. J. Anim. Sci., 31, 745-750.

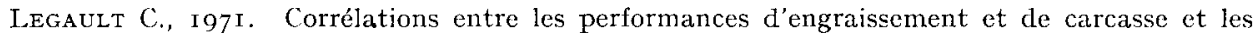
performances d’élevage chez le Porc. Ann. Génét. Sél. anim., 3, I53-160.

Legault C., 1973. Déterminisme génétique de la précocité sexuelle, du taux d’ovulation, et du nombre d'embryons chez la truie primipare : héritabilité, effet d'hétérosis. Joumées Rech. Porcine en France, I47-I54, INRA, ITP Ed., Paris.

Legault C., Dagorn J., 1973. Incidence de l'âge à la première mise-bas sur la productivité de la truic. Journées Rech. Porcine en France, 227-237, INRA, ITP Ed., Paris.

Lerner E. H., Mayer D. T., Lasley J. F., 1957. Early embryonic mortality in strain crossed gilts. Res. Bull. Mo. agric. Exp. Sta. no 629.

Lodge G. A., Mac Pherson R. M., i 961 . Level of feeding cluring early life and the subsequent reproductive performance of sows. Anim. Prod., 3, 19-28.

Martinat Françoise, J.egault C., du Mesnil du Buisson F., Oll.ivier L., Signoret J. P., 1970. Étude des retards de puberté chez la truie. Joumées Rech. Porcine en France, 47-54, INRA, ITP Ed., Paris.

Meacham T. N., Masincupp F, B., 1970. Effect of confinement on reproduction and several blood components in gilts. J. Anim. Sci., 31, 226 (Abstr.).

Du Mesnil du Buisson F., Signoret J. P., i973. L'insémination artificielle porcine vue par un physiologiste. in "l'insémination artificielle porcine", 5-26, ITP Ed., Paris.

Molenat M., Houix Y., Poulenc J., 1974. Contrôles d'engraissement et de carcasses en stations chez les porcins : bilan et réflexions (1967-1973). Bull. Tech. Dép. Génét. anim. (Inst. nat. Rech. Agron. Fr.) $\mathrm{n}^{\circ} 18$.

Nelson R. E., Robison O. W., I976. Effects of postnatal maternal environment on reproduction of gilts. J. Anim. Sci., 43, 71-77.

O'Bannon R. H., Wallace H. D., Warnick A. C., Combs G. E., ig66. Influence of energy intake on reproductive performance of gilts. J. Anim. Sci., 25, 706-710.

Reddy V. B., Lasley J. F., Mayer D. T., I958. Genetic aspects of reproduction in swine. Res. Bull. Mo. agric. Exp. Sta. no 666.

Reutzel L. F., Sumption I.. J., 1968. Genetic and phenotypic relationships involving age at puberty and growth rate of gilts. J. Anim. Sci., 27, 27-30.

Roussead F., 1975. Analyse de quelques aspects phénotypiques et génétiques cles composantes des activités ovarienne et testiculaire chez le porc. Mémoire fin d'étude, E.N.I.T.A., Bordeaux, $52 \mathrm{p}$.

Salmon-Legagneur E., 1970. Étude de quelques facteurs do variation do l'âge et du poids des truies large White au premier wstrus. Joumes Rech. Porcine en France, $4^{\mathrm{T}-4}$, INRA, ITP E.d., Paris. 
Seymour E. W., Speer V. C., Hays V. W., Mangold D. W., Hazen T. E., ig64. Effects of dietary protein level and environmental temperature on performance and carcass quality of growing-finishing swine. J. Anim. Sci, 23, 375-379.

SKJERVOLD H., I975. Comparisons of litter size by use of natural and by artificial mating in pigs. Z. Tievzücht. Zücht Biol., 92, 252-259.

Sprouse W. D., Veum T. L., Mc Fate K. L., Grimes G. A., 1975. A comparison of enclosed confinement and outdoor nursery and growing-finishing systems of swine production. Univ. Miss. Columbia Coll. Agric. Res. Bull. no roog.

Wahlstrom R. C., Fredrikson J. F., Libal G. W., i97i. Effects of housing environment, dietary protein level and sex on performance of growing-finishing swine during winter seasons. J. Anim. Sci., 32, I $138-\mathrm{II}_{4} 2$.

Young L. D., OMtvedt I. T., Johnson R. K., 1974. Relationship of various measures of performance with ovulation rate and number of embryos 30 days after breeding in gilts. $J$. Anim. Sci., 39, $480-487$.

Zimmerman D. R., Spies H. C., Rigor E. M., Self H. L., Casida L. E., i96o. Effect of restricted feeding, cross breeding and season of birth on age at puberty in swine. J. Anim. Sci., I9, $687-694$. 